Revista Luna Azul - APROXIMACIÓN AL CONFLICTO AMBIENTAL URBANO... $\quad$ Página 1 de 8

\title{
APROXIMACIÓN AL CONFLICTO AMBIENTAL URBANO, GENERADO POR LA CONTAMINACIÓN ELECTROMAGNÉTICA NO IONIZANTE EN COLOMBIA
}

\author{
Javier Ignacio Torres $\mathrm{O}$. \\ Ingeniero electricista. MsC \\ Departamento de Física \\ Universidad Tecnológica de Pereira \\ oscuro@utp.edu.co \\ Luz Elena Agudelo \\ Abogada, M.Sc. \\ Profesor Catedrático \\ Universidad Libre seccional Bogotá \\ Magíster en Derecho Ambiental \\ Especialista en Derecho Urbano \\ luzelenaas@hotmail.com \\ Martha Cecilia Ochoa Osorio \\ Administradora del Medio Ambiente \\ Especialista en Gestión Ambiental Local \\ Universidad Tecnológica de Pereira \\ libelula@utp.edu.co \\ Manizales, 2007-11-30 (Rev. 2007-12-20)

\section{RESUMEN} \\ En este trabajo se presentan las bases fundamentales requeridas para la aproximación \\ y entendimiento del conflicto ambiental urbano generado por las limitantes técnico- \\ normativas en lo referente a la legislación de la exposición de la ciudadanía a \\ contaminación electromagnética no ionizante (CEM NI). Se realiza un acercamiento a la \\ situación que se deriva de la generación de esta contaminación, sus implicaciones \\ jurídicas y ambientales dentro de las relaciones sociedad-naturaleza, se exponen \\ algunas recomendaciones para mejorar la situación respecto a la CEM NI y el posible \\ conflicto que estas pueden suscitar.
}

\section{PALABRAS CLAVE}

Ambiental, conflicto, contaminación, electromagnética, jurídico, norma.

APPROXIMATION OF THE URBAN ENVIRONMENTAL CONFLICT, GENERATED BY THE ELECTROMAGNETIC NON IONIZING POLLUTION IN COLOMBIA

\begin{abstract}
This work shows the fundamental basis for the approximation and understanding of urban environmental conflicts generated by the technical normative limitations in regards to legislation corresponding to the citizen exposure to non ionizing electromagnetic pollution (NI EMP). An approach to the situation related to the generation of said pollution, its legal and environmental implications within societynature relationships, and some recommendations oriented to improve the current state of NI EMP and the conflicts being generated as a byproduct are carried out.
\end{abstract}


Revista Luna Azul - APROXIMACIÓN AL CONFLICTO AMBIENTAL URBANO... $\quad$ Página 2 de 8

\section{KEY WORDS}

Environmental, conflict, pollution, electromagnetic, legal, regulation.

\section{INTRODUCCIÓN}

La contaminación electromagnética no ionizante se da como subproducto de las actividades de generación, transmisión y distribución de energía eléctrica, para uso doméstico e industrial y también de actividades de telecomunicación. Estas actividades vistas como procesos inherentes a las actividades cotidianas de la sociedad, dejan al hombre inmerso en un ambiente electromagnético artificial, que puede afectar al individuo y a la población en general [1][2]. Esta afectación se relaciona con el riesgo antrópico que se deriva del uso o aplicación inadecuada de tecnologías, que se manifiesta cuando se exceden los límites recomendados por la normatividad ambiental, los cuales pueden generar conflictos ambientales urbanos [3]. Dado este panorama, en Colombia se han generado normas de carácter jurídico-técnico que están dirigidas a la protección de la población que a pesar de estar consagradas para proteger al hombre [4], presentan falencias en su conformación y para su aplicación, lo que puede generar escenarios propicios para la incubación de un nuevo tipo de conflicto ambiental urbano. La principal fuente para estos conflictos es el desconocimiento de este tipo de contaminación por la comunidad, dado que no es perceptible a simple vista bajo condiciones normales. Para poder acercarse a este tema se deben clarificar unos conceptos básicos y analizar la legislación vigente y los métodos de aplicación.

\section{FUNDAMENTO TEÓRICO}

Se debe recordar que de forma permanente, incluso antes de nacer, estamos expuestos a la acción de campos electromagnéticos no lonizantes (CEM NI) naturales, entendiendo como tales aquellos que están ligados al planeta tierra y los de origen cósmico. Desde la perspectiva del riesgo, corresponde a un factor de amenaza natural que está determinada por los procesos intrínsecos a la dinámica de la tierra.

Para ampliar sobre la fundamentación técnica referente a conceptos como campo electromagnético, contaminación electromagnética, los posibles contaminantes y efectos de este tipo de energía radiante, remitirse a [3].

\subsection{Conflicto Ambiental [5]}

El término conflicto proviene de la palabra en latín "conflictus" que significa choque. Hablar de él es referirse a una situación en la que un actor(es) se encuentra en oposición conciente con otro(s) actor(es), debido a que persiguen objetivos contrarios, lo que los coloca en extremos antagónicos, en situación de enfrentamiento, confrontación y lucha. El conflicto ambiental hace referencia a procesos sociales suscitados por el desacuerdo que genera la apropiación, distribución y utilización de los recursos naturales y a la movilización y denuncia contra los causantes de los daños ecológicos (1) . Francisco Sabatini (2), utiliza la categoría de conflicto ambiental para referirse a los que se dan en torno a la distribución de las denominadas "externalidades" o "efectos externos" derivados de los usos del suelo, es decir, de nuevas actividades que se desarrollan en un lugar. Para este autor los conflictos ambientales se han derivado de los modelos de desarrollo, evidencian la crisis de la modernidad y los ubica en este contexto social y político del modelo económico neoliberal que privatiza el lucro económico y socializa los problemas ambientales.

\subsubsection{Tipos de conflictos ambientales}

Los conflictos expresan las relaciones entre naturaleza y cultura. Su manifestación concreta se da en un territorio, para este caso la ubicación de infraestructura eléctrica y de telecomunicaciones al interior de una zona residencial que esté afectando a la población asentada en esa zona puede ser un escenario de conflicto latente. En ese sentido, aunque las causas estructurales de los conflictos pueden estar dadas por relaciones macroeconómicas globales, los efectos de los conflictos ambientales se dan en el ámbito local. De acuerdo a lo expuesto por Quintana, el abordaje de un conflicto 
implica una caracterización de éste. Dependiendo de la causa que determina el tipo de conflicto ambiental presente en la zona, se identificará la elección y formulación del procedimiento más adecuado en el manejo del mismo. Los tipos de conflicto ambiental más frecuentes, de acuerdo a lo expuesto por [5], son cuatro. De éstos los causantes del posible conflicto ambiental por contaminación electromagnética no ionizante son:

- Conflictos por problemas de datos: en los casos en los que falta la información necesaria para la toma de decisión, cuando las personas están mal informadas, o cuando está en discusión la relevancia de los datos con que se cuenta o su interpretación. Hay conflictos que pueden en gran parte superarse con el suministro de información adecuada y confiable. Sin embargo, es usual que la información sea manipulada en función de los intereses de las partes en disputa, generando imágenes públicas que después es muy difícil remover (3) . Es evidente que para el caso de conflictos ambientales asociados a la contaminación electromagnética no ionizante, la principal causa para que se presente está determinada por la falta de entes acreditados, en el caso colombiano, que puedan hacer mediciones confiables de los niveles de este tipo de contaminación y que la comunidad que es afectada tenga acceso a la información para poder sustentar sus quejas y reclamos y pueda generar acciones en pro de corregir los problemas causados.

- Conflictos por divergencia de intereses: tienen su origen en la disputa por intereses realmente divergentes o que han sido interpretados como tales. Estos conflictos surgen frecuentemente cuando los participantes del mismo exigen a los otros renunciar a sus intereses, a fin de imponer los propios. La razón del conflicto puede ser, entonces, tanto de naturaleza sustancial (dinero, tiempo, recursos) de procedimiento (¿cómo se resolverá el conflicto?) como psicológica (percepción de confianza, honestidad, respeto). Condición para la solución de este tipo de conflictos es que la ésta satisfaga una proporción suficiente de los intereses de todos los participantes en estas tres áreas. A este tipo de conflicto se le denomina también conflicto in-situ, porque suscitan un determinado impacto ambiental entre los actores de una localidad, sobre todo intereses económicos y relativos a la salud y calidad de vida. Especialmente importantes son los conflictos provocados por actividades productivas que afectan a una comunidad y al medio ambiente local.

Los otros dos tipos de conflicto ambiental son: conflictos por divergencia de valores o de enfoque y conflictos por problemas estructurales.

\section{ELEMENTOS DE ANÁLISIS DEL CONFLICTO POR CEM NI.}

3.2 Análisis de las relaciones Sociedad-Naturaleza: todo proceso de interacción entre la sociedad y la naturaleza genera, inevitablemente, transformaciones y cambios. Estos cambios y transformaciones se llaman impactos. Es una relación dialéctica, la naturaleza se transforma por la acción humana, así mismo la sociedad debe asumir cambios.

3.3 Impacto Ambiental: los procesos de transformación dados en la naturaleza pueden ser positivos y/o negativos. Esta valoración es antrópica, es decir, sólo la sociedad lo define en términos de valoración relacionados con la afectación. Cuando la sociedad asume concientemente que dichos impactos alteran la vida cotidiana de las personas y esto se hace evidente se dice que se presenta un problema ambiental. Un problema ambiental es la manifestación de ese impacto. Por ejemplo, para el caso en cuestión sería la contaminación electromagnética no ionizante.

3.3.1 Riesgo: se habla de riesgo cuando existe la probabilidad de afectaciones o pérdidas a la sociedad o algún elemento natural que se encuentre expuesto a la manifestación de una amenaza. El riesgo por contaminación electromagnética puede categorizarse como un riesgo antrópico ya que está determinado por la acción humana asociada al uso de la tecnología. El riesgo puede convertirse en un conflicto ambiental dada su categoría social, es decir, en el proceso de configuración de un escenario de riesgo las amenazas no representan por sí mismas una condición de riesgo como tal, son las relaciones entre un factor amenazante y una población vulnerable la que lo 
define. Desde la gestión del riesgo se habla de una gestión correctiva y una gestión prospectiva. La gestión correctiva hace alusión a las acciones que deben encaminarse para compensar y/o mitigar el riesgo que ya existe, por ejemplo ¿qué hacer ante la presencia de antenas que generan contaminación electromagnética en una zona residencial de influencia directa? Una acción correctiva o de mitigación sería la reubicación o cualquier medida que minimice la exposición de los elementos vulnerables o expuestos. La gestión prospectiva en cambio, son acciones a mediano y largo plazo que están orientadas a prevenir la aparición del riesgo en nuevos escenarios. Por ejemplo para este caso, son medidas relacionadas con la reglamentación, aplicadas a los procesos de ordenamiento territorial y desarrollo urbano que prevean las implicaciones a futuro de la localización de infraestructura eléctrica y de telecomunicaciones dentro del territorio que se haya definido como zona de expansión urbana.

\section{REGLAMENTACIÓN NACIONAL}

La legislación vigente con relación a las restricciones que se contemplan en materia de protección a los usuarios de los sistemas de distribución de energía, se encuentra en el reglamento Técnico para Instalaciones Eléctricas (RETIE) [6]. Y para los sistemas de telecomunicaciones, se cuenta con los límites permisibles de exposición a radiaciones de radiofrecuencia (RF) [7], que tienen como finalidad proteger la salud de la comunidad y el ambiente de los efectos nocivos de la Contaminación Electromagnética no ionizante, no obstante, el desconocimiento de la normatividad en cuanto a los mecanismos de protección ambiental que tiene la población, hace que no se aplique rigurosamente. Igualmente, las autoridades que tienen la responsabilidad de llevar a cabo la regulación, ejecución, control y vigilancia ambiental como el Sistema Nacional Ambiental (SINA) son laxas en el cumplimiento de sus funciones, y otras veces se requieren cambios o ajustes en la implementación de la norma.

\subsection{Frecuencia extremadamente baja [6]}

En este rango se cuenta con un aparte del RETIE (Reglamento Técnico para Instalaciones Eléctricas) que define requisitos para intensidad de campo eléctrico y densidad de flujo magnético para frecuencias comprendidas entre 25 a $1000 \mathrm{~Hz}$, en zonas donde pueda permanecer el público.

\begin{tabular}{|c|c|}
\hline Intensidad de campo eléctrico $\mathrm{E}(\mathrm{kV} / \mathrm{m})$ & Densidad de flujo magnético $\mathrm{B}(\mathrm{mT})$ \\
\hline \hline 10 & 0.50 \\
\hline
\end{tabular}

Tabla 1: RETIE. Niveles de referencia para campos electromagnéticos. 2005

Y se agrega: debe entenderse que ningún sitio donde pueda estar expuesto el público debe superar estos valores. Para líneas de transmisión estos valores no deben ser superados dentro de la zona de servidumbre y circuitos de distribución a partir de distancias de seguridad (2,3 m horizontal). Pero el RETIE en el capítulo II define estas distancias de seguridad para contacto y no se define explícitamente si cubren el riesgo por radiación electromagnética. Este reglamento para CEM presenta inconsistencias que se deben tratar con cuidado.4.2 Para Radio Frecuencia [7]

La restricción para radiofrecuencia (RF) se da mediante resolución del Ministerio de Comunicaciones, número 001645 del 29 de julio de 2005, que reglamenta los límites permisibles de exposición a radiaciones de RF basado en recomendación de la Unión Internacional de Telecomunicaciones, (UIT-T K.52) "Orientación sobre el cumplimiento de los límites de exposición de las personas a los campos electromagnéticos", vigente desde 2005.

\section{MECANISMOS JURÍDICOS PARA LA APLICACIÓN DE LA REGLAMENTACIÓN NACIONAL}

Colombia ha sido un país pionero en cuanto a normatividad ambiental se refiere, esto se manifiesta con la expedición del Código Nacional de Recursos Naturales Decreto 2811 de 1974, como respuesta temprana a la Conferencia de Estocolmo, realizada en 
1972, en donde se expresa por vez primera el clamor de la comunidad internacional para que los Estados tomaran conciencia ambiental; La Asamblea Constituyente que dio lugar a la Constitución Política de 1991, realizó un profundo análisis de la necesidad de incluir el ambiente como eje fundamental del Estado colombiano, pudiendo afirmar la Corte Constitucional [8], que ésta es una constitución no solamente económica, política, cultural y social, sino también ecológica, toda vez que cuenta con un abordaje a lo largo y ancho de su texto de criterios ambientales. Como reglamentación de la Constitución del 91, se expidió la Ley 99/93 y posteriormente se promulga la Ley 388 de 1997 Ley de Desarrollo Territorial, normas éstas que destacan la protección del ambiente y el derecho que tienen todos los habitantes del territorio nacional a gozar de un ambiente sano que garantiza la vida en condiciones de calidad.

Las reglamentaciones con que se cuenta concretamente respecto de la contaminación electromagnética son:

Nuestro ordenamiento jurídico ha previsto estos límites de contaminación permisibles, en aras de lograr el desarrollo sostenible, sin embargo, superar tales límites puede generar conflictos ambientales urbanos, que evidencian la ineficacia de la norma, lo que refleja flagrante violación al derecho a gozar de un ambiente sano, contenido en el artículo 79 de la Constitución Política, por lo que es necesario hacer ajustes a fin de lograr el objetivo de la normatividad, cual es el bienestar común.

Las adecuaciones normativas necesarias para el caso de la CEM NI, tienen por objeto evitar los riesgos que científicamente no se han podido descartar, este riesgo las justifica y puede ser tratado desde el punto de vista jurídico a partir del principio de precaución o cautela, que surgió como uno de los 27 principios de la Declaración de Río de Janeiro de 1992 sobre Medio Ambiente y Desarrollo, incorporado en la Ley 99 de 1993 como uno de los que orienta la política ambiental nacional, cuya exequibilidad fue declarada por la Honorable Corte Constitucional mediante sentencia C-528 de 1994, de tal forma que su aplicación es plena en Colombia, bajo los presupuestos contenidos en la sentencia C-293 de 2002 [BILIO] del mismo alto tribunal, en donde se le hizo un profundo análisis. El principio de cautela tiene como propósito evitar riesgos antes que aplicar remedios al mal causado, es decir, que si existe la probabilidad de que ocurra un daño grave e irremediable, aunque no se tenga certeza científica de su ocurrencia, los Estados deben aplicar todas las medidas tendentes a evitarlo, especialmente porque los daños ambientales no tienen consecuencias inmediatas, es decir, opera el fenómeno de la latencia, toda vez que los efectos nocivos de la contaminación, en este caso la electromagnética no ionizante, posiblemente se reflejen a largo plazo.

Como se ha señalado, el Estado ha regulado la contaminación electromagnética, implementando límites con el fin de evitar daños en la salud de las personas y en el ambiente (ver RETIE), tales disposiciones carecen de eficacia, toda vez que no se han establecido mecanismos de verificación por parte de las entidades públicas, ni procedimientos que permitan el cumplimiento de su objetivo.

La Constitución de 1991, la Ley 99 de 1993, y las distintas disposiciones ambientales, han consagrado como mecanismo de protección del medio ambiente la necesidad y obligatoriedad de la participación de la comunidad en las distintas decisiones que en materia ambiental se adopten, de conformidad con el nuevo orden de democracia participativa implementado por el Estado colombiano, en primer lugar para invocar la protección de sus derechos y, en segundo lugar, para procurar que lo consagrado por la ley se cumpla y se generen alternativas legales a las propuestas gubernamentales.

Como mecanismos de participación, concretamente en materia ambiental, se destacan en nuestro ordenamiento jurídico: el derecho de información, la consulta obligatoria a comunidades indígenas y negras, el derecho de intervención, la celebración de audiencias públicas y las veedurías ciudadanas, los que deberán combinarse con la educación ambiental, en razón a que con ésta se genera conocimiento, conciencia y se incentiva la concurrencia de la comunidad en la toma de decisiones tanto administrativas como legislativas que las afectan directamente. Los citados mecanismos de participación en mayor grado tienen una finalidad preventiva, porque lo que pretenden es que la adopción de las decisiones estatales, concretamente en materia ambiental, estén sometidas al escrutinio de la población que en últimas es la que resultará afectada, sin embargo, por el desconocimiento imperante de la comunidad en general, pueden surgir conflictos ambientales que amenazan o lesionan derechos constitucionales, siendo necesaria la adopción de otros instrumentos cuyo propósito es 
Revista Luna Azul - APROXIMACIÓN AL CONFLICTO AMBIENTAL URBANO... Página 6 de 8

hacer cesar la amenaza a los derechos, su reconocimiento y restablecimiento.

En relación con estos instrumentos ya no preventivos sino correctivos, de carácter constitucional, se resalta en primer lugar la acción popular, consagrada en el artículo 88 de la Constitución Política, reglamentado por la Ley 472 de 1998, que garantiza la defensa y protección de los derechos colectivos, dentro de los que se encuentra el ambiente, teniendo como objetivo hacer cesar la amenaza y restituir las cosas a su estado anterior, siendo la principal a la hora de que se restablezcan los derechos de las personas en lo que a medio ambiente se refiere.

La acción de grupo, igualmente contemplada y reglamentada por las mismas normas que la acción popular, tiene como finalidad la indemnización de los perjuicios que se le hayan producido a un número determinado de personas, con ocasión de una actividad derivada de la vulneración de cualquier derecho, incluido el ambiente sano.

La acción de tutela a la que hace referencia el artículo 86 de la Constitución Política, busca la protección inmediata de los derechos fundamentales, opera también como garante del derecho a gozar de un ambiente sano, toda vez que aunque este no sea considerado un derecho fundamental. La Corte Constitucional ha señalado en reiterada jurisprudencia que por conexidad con otros derechos que sí tienen tal entidad, éste puede ser protegido a través de este medio, que se destaca por ser expedito, sumario y preferencial.

Por otro lado se encuentra la acción de cumplimiento, que tiene como propósito la efectividad de las normas con rango de ley, de los actos administrativos de carácter general y, excepcionalmente, de los actos administrativos de carácter particular. Contemplada en el artículo 87 de la Constitución Política y reglamentada por la Ley 393 de 1997, con importantes antecedentes en las leyes 99 de 1993 y 388 de 1997, para el caso que nos ocupa, tiene especial relevancia, toda vez que es a través de este instrumento que cualquier persona podrá acudir ante la autoridad judicial y exigir el cumplimiento por parte de la autoridad renuente. Si se está discutiendo la efectividad de las normas sobre contaminación electromagnética, que genera conflictos ambientales urbanos en torno al desconocimiento del derecho constitucional a gozar de un ambiente sano, es la acción de cumplimiento la llamada a ser utilizada, con las restricciones que se han impuesto para su ejercicio, a fin de que se verifique el bien común como objetivo de la expedición de las distintas normas.

Otras acciones, ya no de carácter constitucional, sino de orden legal, que son instrumentos adecuados para la exigencia de los derechos, particularmente el de gozar de un ambiente sano con la correspondiente indemnización de perjuicios, son las acciones judiciales de reparación directa, cuando el sujeto dañino sea el Estado; la acción de responsabilidad civil extracontractual por daño ambiental, cuando el litigio se presente entre particulares; y la acción penal, cuando se trate de la comisión de delitos ambientales.

\section{MANEJO DEL CONFLICTO}

Desde la teoría del conflicto, varios autores afirman que éstos son necesarios, ya que generan procesos de transformación cultural en la medida que la sociedad hace conciencia de las afectaciones y problemas ambientales que alteran la vida y su normal desarrollo. En ese sentido las formas de abordar un conflicto se da entre las partes que sienten afectados sus intereses. Se habla de un manejo tradicional o adversarial cuando las acciones se centran en acciones jurídicas. Se habla de un manejo alternativo cuando las partes deciden asumir el conflicto y darle una salida que comprometa a los intereses de los mismos, la mediación, la conciliación, la planificación y gestión asociada son algunas formas de manejo alternativo de los conflictos ambientales.

\section{CONCLUSIONES}

- En Colombia existe todo un contenido jurídico de mecanismos para la protección del ambiente, pero dada la carencia de entes acreditados por La Superintendencia de Industria y Comercio (SIC), en la realización de mediciones del nivel de intensidad de los campos electromagnéticos a los que está expuesta la población que permitan la verificación de la norma, se presenta 
Revista Luna Azul - APROXIMACIÓN AL CONFLICTO AMBIENTAL URBANO... $\quad$ Página 7 de 8

un posible conflicto ambiental urbano generado por conflicto de datos.

- Se debe hacer una adecuación de las normas apelando a principios como el de cautela o prevención, para que se pueda realizar la protección de la salud y del derecho a gozar de un ambiente sano. Dado que las reglamentaciones carecen de efectividad y sumado al desconocimiento imperante en la comunidad estas normas se deben incorporar en los procesos de ordenamiento territorial.

- Es marcada la tendencia a la generación de conflictos ambientales, por lo que se debe reglamentar y capacitar personal en aras de evitar y solucionar tales conflictos con mecanismos alternativos como la conciliación extrajudicial, en derecho o en equidad.

- Se debe incentivar la formación de grupos interdisciplinarios para afrontar el estudio de la contaminación EM-NI en Colombia.

\section{BIBLIOGRAFÍA}

[1] General approach to protection against non-ionizing radiation. Health Physics Society. International Commission On Non-Ionizing Radiation Protection (ICNIRP). P.539-548. 2002.

[2] Torres, J. y Alzate, L. Efectos de las radiaciones electromagnéticas no ionizantes en sistemas biológicos. Revista medica del Risaralda. Vol 12 № 2 Noviembre de 2006.

[3] Torres, J y Ochoa, M. Criterios tecnicoambientales para el análisis del riesgo por contaminación electromagnéticas no ionizantes en Colombia. Revista Luna Azul. $N^{\circ} 24$ enero-junio 2007.

[4] Llamosa, L. E. y Torres, J. Fundamentos para una propuesta de norma técnicoambiental sobre radiaciones electromagnéticas de frecuencia extremadamente baja (FEB), SCIENTIA ET TÉCNICA. Octubre de 2003. No 22 p.143- 148.

[5] Quintana, A. El conflicto socioambiental y estrategias de manejo. Presented at Congreso???. Meeting. [Online]. Available: http://www.asocars.org.co/archivos/grupos/Cuencas/D\%C3\%ADa\%2015\% 20diciembre/Ana\%20Patricia\%20CONFLICTO\%20Y\%20RESLUCION.doc

[6] Reglamento Técnico Para Instalaciones Eléctricas. (RETIE). MINISTERIO DE MINAS Y ENERGÍA. Bogotá, 2005.

[7] RESOLUCIÓN NÚMERO 001645 MINISTERIO DE COMUNICACIONES REPÚBLICA DE COLOMBIA. Julio 29. 2005, por la cual se reglamenta el Decreto 195 de 2005 .

[8] Sentencia T-411 de 1992. Corte Constitucional.

\section{Bibliografía adicional}

- SANTOdOMingo, M. Lecturas sobre derecho del medio ambiente. Tomo IV. Universidad Externado de Colombia. Octubre 2003 p. 457- 482.

- Parlamento Europeo. Notas informativas N•297.563/2001 y 297.574/2001.

- Informe final $\vee$ congreso nacional del medio ambiente, Grupo de trabajo 13 Campos Electromagnéticos. Nov 2000.

- Informe final VI congreso nacional del medio ambiente, Grupo de trabajo sobre Campos Electromagnéticos. Febrero 2003. Madrid España.

- Encuentro regional sobre los campos electromagnéticos, Latinoamérica y el caribe, Lima-Perú, marzo 2001.

\section{NOTAS}


Revista Luna Azul - APROXIMACIÓN AL CONFLICTO AMBIENTAL URBANO... $\quad$ Página 8 de 8

1. CORANTIOQUIA. Manejo de Conflictos Ambientales. Primera edición. Medellín, Colombia. 2001. p. 9.

2. SABATINI, Francisco y SEPÚLVEDA L., Claudia. Conflictos Ambientales, entre la globalización y la sociedad civil. Publicaciones CIPMA. Santiago de Chile, 2002. p. 50-54.

3. MARTíNEZ GONZÁLEZ, Alfonso. Métodos Alternativos en Manejo de Conflictos. Aplicaciones en materia ambiental. México: SEMARNAP, PNUD. 1997. p. 17.

Close Window 